\title{
Characterization of a Method for Inverse Heat Conduction Using Real and Simulated Thermocouple Data
}

\author{
Michelle E. Pizzo ${ }^{\mathrm{a}}$ \\ Old Dominion University, Norfolk, VA 23529, USA \\ Information Technology Infrastructure Branch, NASA Langley Research Center, Hampton, VA 23681, USA \\ David E. Glass ${ }^{\mathrm{b}}$ \\ Structural Mechanics and Concepts Branch, NASA Langley Research Center, Hampton, VA 23681, USA
}

\begin{abstract}
It is often impractical to instrument the external surface of high-speed vehicles due to the aerothermodynamic heating. Temperatures can instead be measured internal to the structure using embedded thermocouples, and direct and inverse methods can then be used to estimate temperature and heat flux on the external surface. Two thermocouples embedded at different depths are required to solve direct and inverse problems, and filtering schemes are used to reduce noise in the measured data. Accuracy in the estimated surface temperature and heat flux is dependent on several factors. Factors include the thermocouple location through the thickness of a material, the sensitivity of the surface solution to the error in the specified location of the embedded thermocouples, and the sensitivity to the error in thermocouple data. The effect of these factors on solution accuracy is studied using the methodology discussed in the work of Pizzo, et. al. ${ }^{1}$ A numerical study is performed to determine if there is an optimal depth at which to embed one thermocouple through the thickness of a material assuming that a second thermocouple is installed on the back face. Solution accuracy will be discussed for a range of embedded thermocouple depths. Moreover, the sensitivity of the surface solution to (a) the error in the specified location of the embedded thermocouple and to (b) the error in the thermocouple data are quantified using numerical simulation, and the results are discussed.
\end{abstract}

$\begin{array}{ll} & \\ 1-\mathrm{D} & =\text { One-dimensional } \\ c_{p} & =\text { Specific heat capacity, } \mathrm{J} /(\mathrm{kg}-\mathrm{K}) \\ \mathrm{DHCP}, \mathrm{IHCP} & =\text { Direct (respectively, inverse) heat conduction problem } \\ e_{q}(t), e_{T}(t) & =\text { Error in heat flux (respectively, temperature), } \% \\ k & =\text { Thermal conductivity, } \mathrm{W} /(\mathrm{m}-\mathrm{K}) \text { or } \mathrm{W} /(\mathrm{cm}-\mathrm{K}) \\ \mathrm{L} & =\text { Thickness of material specimen } \\ m & =\text { Superscript denoting the time step count } \\ n & =\text { Subscript denoting the nodal count } \\ N_{D}, N_{I} & =\text { Number of nodes for the DHCP }(\text { respectively, IHCP) } \\ q & =\text { Heat flux, W/m }{ }^{2} \text { or W/cm }{ }^{2} \\ T & =\text { Temperature, } \mathrm{K} \\ t & =\text { Time, } \mathrm{s} \\ x_{n} & =\text { Spatial location, } \mathrm{m} \text { or } \mathrm{cm} \\ \alpha & =\text { Thermal diffusivity }=k / \rho c_{P}, \mathrm{~m}^{2} / \mathrm{s} \\ \Delta t & =\text { Time step size, } \mathrm{s} \\ \Delta x & =\text { Cell size, } \mathrm{m} \text { or } \mathrm{cm} \\ \in & =\text { Symbol denoting the phrase is an element of } \\ \rho & =\text { Mass density, } \mathrm{kg} / \mathrm{m}^{3} \text { or } \mathrm{kg} / \mathrm{cm}^{3}\end{array}$

${ }^{a}$ Ph.D. Candidate, Pathways Student Trainee

${ }^{b}$ AIAA Associate Fellow 


\section{Introduction}

$\mathrm{W}$ hile traveling at hypersonic speeds, flight vehicles encounter high heating resulting in high surface temperature. There is an interest in knowing these surface values particularly in the fields of materials, structures, and aerothermodynamics research. Unfortunately, it is usually either impractical or impossible to instrument the external surface of high-speed vehicles due to the aerothermodynamic heating. Temperature histories are instead measured internally to the surface by embedding thermocouples or thermocouple plugs through the thickness of the vehicle skin or thermal protection system. The measurements can then be used to solve an inverse heat conduction problem (IHCP) to estimate surface temperature and heat flux. The inverse problem is ill-posed. Therefore, the solution is extremely sensitive to measurement errors, i.e., small errors in the data yield large errors in the solution ${ }^{2-6}$.

The study of inverse heat conduction started in the late 1950s with work published by Shumakov ${ }^{7}$ and $\mathrm{Stolz}^{8}$ in 1957 and 1960, respectively. In the years since, numerous methods for solving both linear (constant thermophysical properties) and nonlinear (temperature-dependent thermophysical properties) one-dimensional (1-D) transient inverse problems have emerged from researchers in the field such as $\mathrm{Beck}^{3,9,10,11}$, $\mathrm{Murio}^{6}$, Weber ${ }^{2}$, and Carasso ${ }^{5}$, among others.

In 2016, Pizzo, et. al., ${ }^{1}$ estimated surface temperature and heat flux histories of high-temperature carbon/carbon materials using a 1-D inverse method (defined within the body of paper) with temperature-dependent thermophysical properties (nonlinear problem) assuming negligible in-plane temperature gradients. The study was motivated by Frankel, et. al. , $^{42,13}$. In his work, Frankel solved the 1-D transient heat conduction problem using noisy data measured at internal depths $x_{1}$ and $x_{2}$ where $x_{1}<x_{2}$. He reduced noise in the data with a global low-pass Gaussian filter by defining a cutoff frequency using discrete Fourier transforms of the data. Frankel then approximated the heating/cooling rate using finite differences and numerically integrated the integral relationship between temperature and heat flux to obtain an analytical heat flux solution at depth $x_{2}$. The surface temperature and heat flux were approximated from the measured temperature and calculated heat flux at depth $x_{2}$ using finite differences and Taylor series reconstruction. This method yielded accurate results.

The methodology in Pizzo, et. $a l^{1}$ used Frankel's procedures as a guide, but made two slight modifications. First, local windowed-sinc filters were used to reduce noise in the measured data. Second, a technique developed by Carasso ${ }^{5}$ was used to solve the inverse problem. In this technique, the 1-D heat conduction equation is first written as a firstorder system with temperature and heat flux as the unknowns, and is then discretized using forward differencing in space and central differencing in time. The methodology in [1] was validated using data provided by thermal vacuum chamber radiant heating tests (c.f. Blosser ${ }^{14}$ ). Pizzo demonstrated accuracy in estimating temperature histories using comparisons with test results (estimated solutions had error in the range of $-5 \%$ to $9 \%$ ). The estimated heat flux histories however had significantly larger error than temperature (estimated solutions had error in the range of $-30 \%$ to $10 \%)$. The larger errors were expected, because for the methodology used, the rate of convergence with heat flux with mesh size is one order less accurate than temperature.

The purpose of this paper is to expand upon the work in [1] by characterizing the selected method for inverse heat conduction. For inverse methods, because small errors in the data yield large errors in the solution, accuracy in the estimated surface temperature and heat flux is dependent on several factors. Factors include the embedded thermocouple depth through the thickness of a material, the sensitivity of the surface solution to the error in the specified location of the embedded thermocouple, and the sensitivity of the solution to the error in thermocouple data. The effect of these factors on solution accuracy is presented in this paper.

The inverse methodology is summarized in Section II, and the characterization of the method is discussed in Section III. Concluding remarks are discussed in Section IV.

\section{Methodology Summary}

The transient temperature distribution in a material is governed by the heat conduction equation. Assuming that the in-plane temperature gradients are negligible, the heat conduction equation reduces to its 1-D form:

$$
\rho c_{p}(T) \frac{\partial T}{\partial t}=\frac{\partial}{\partial x}\left(k(T) \frac{\partial T}{\partial x}\right)
$$

where $\rho, c_{p}$, and $k$ are the density, specific heat, and thermal conductivity of the material, respectively. The initial temperature distribution through the thickness of the material is specified at time $t=0$ :

$$
T(x, 0)=T_{i}(x)
$$


Boundary conditions at the outer surfaces $(x=0, L)$ are required to solve Eq. (1). For the classical initial-boundary value problem, referred to in this work as the direct heat conduction problem (DHCP), the boundary conditions are in the form of specified temperature or heat flux ${ }^{3}$. In situations where the boundary values are unknown, an inverse $\mathrm{B}^{3,4,12}$ problem must be solved using known internal temperatures at two depths, denoted by $x_{1}$ and $x_{2}$. The source of the internal temperatures is typically data from a set of embedded thermocouples as illustrated in Figure 1.

In [1], the surface temperature and heat flux histories at $x=L$ are estimated from two internal temperature measurements, $T_{1}$ and $T_{2}$, using a fourstep process. The four-step process includes filtering noise from the measured data, solving for temperature histories in the region between depths $x_{1}$ and $x_{2}$ where $0 \leq x_{1}<x_{2}<L$, solving for the heat flux history at depth, $x_{2}$, and solving for the temperature and heat flux histories in the region extending from depth $x=x_{2}$ to $x=L$.

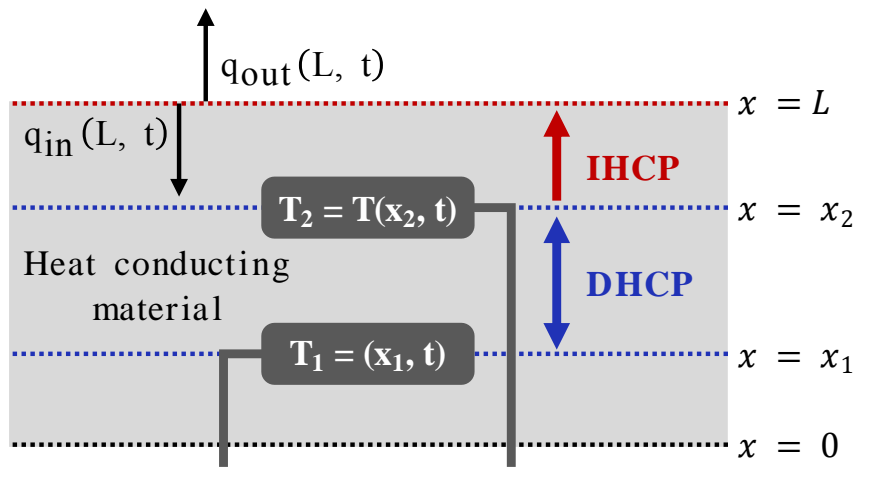

Figure 1. Illustration of the direct and inverse problems and the embedded thermocouples, $T_{1}$ and $T_{2}$.

\section{Solving for Temperature Histories Between Depths $x_{1}$ and $x_{2}$ (Direct Problem)}

The region between thermocouples embedded at depths $x_{1}$ and $x_{2}$ is treated as the DHCP with initial and boundary values given by measured data, i.e.

$$
\begin{aligned}
& T(x, 0)=T_{0}, \quad x_{1} \leq x \leq x_{2} \\
& T\left(x_{1}, t\right)=T_{1}, \quad t>0 \\
& T\left(x_{2}, t\right)=T_{2}, \quad t>0 .
\end{aligned}
$$

Equation (1) is discretized by dividing the domain into a uniform mesh of $N_{D}$ total nodes with cell size of $\Delta x=$ $\left(x_{2}-x_{1}\right) /\left(N_{D}-1\right)$. In the mesh, node $n=1$ is at depth $x=x_{1}$ and node $n=N_{D}$ is at depth $x=x_{2}$. Letting $T_{n}^{m}$ denote the temperature of node $n$ at time $t_{m}=m \Delta t$, the temperature of node $n$ at time $t_{m+1}=(m+1) \Delta t$ is obtained using a vertex-based finite volume method with Crank-Nicholson time marching:

$$
\frac{T_{n}^{m+1}-T_{n}^{m}}{\Delta t}=\frac{\alpha_{n-1, n}^{m}}{2 \Delta x^{2}}\left(T_{n-1}^{m}-T_{n}^{m}+T_{n-1}^{m+1}-T_{n}^{m+1}\right)+\frac{\alpha_{n, n+1}^{m}}{2 \Delta x^{2}}\left(T_{n+1}^{m}-T_{n}^{m}+T_{n+1}^{m+1}-T_{n}^{m+1}\right)
$$

where $\alpha_{n-1, n}^{m}\left(\alpha_{n, n+1}^{m}\right)$ denotes the average of the temperature-dependent thermal diffusivity evaluated at temperatures $T_{n-1}^{m}$ and $T_{n}^{m}\left(T_{n}^{m}\right.$ and $\left.T_{n+1}^{m}\right)$. Since $T_{1}^{m+1}=T\left(x_{1},(m+1) \Delta t\right)$ and $T_{N_{D}}^{m+1}=T\left(x_{2},(m+1) \Delta t\right)$ are known from the filtered data, Eq. (4) yields temperature histories at nodes $n \in\left[2, N_{D}-1\right]$, or equivalently, in the domain $x \in\left[x_{1}, x_{2}\right]$.

\section{Solving for the Heat Flux History at Depth $\boldsymbol{x}_{\mathbf{2}}$ (Direct Problem)}

Let $q_{N_{D}}^{m}$ denote the heat flux entering the domain at node $N_{D}$ at time $t_{m}=m \Delta t$. The heat flux entering the domain at node $N_{D}$ at time $t_{m+1}=(m+1) \Delta t$ is treated as an unknown and is obtained using a consistent approach based upon an energy balance for the half-call $x \in\left[x_{N_{D}}-\Delta x / 2, x_{N_{D}}\right]$ combined with Crank-Nicholson time marching:

$$
\text { Node } N_{D}: q_{N_{D}}^{m+1}=k_{N_{D}}^{m}\left[\frac{T_{N_{D}}^{m}-T_{N_{D}-1}^{m}+T_{N_{D}}^{m+1}-T_{N_{D}-1}^{m+1}}{2 \Delta x}\right]+\rho c_{P_{N_{D}}}^{m}\left(\frac{\Delta x}{2}\right)\left[\frac{T_{N_{D}}^{m+1}-T_{N_{D}}^{m}}{\Delta t}\right]
$$

where $k_{N_{D}}^{m}$ and $c_{p_{N_{D}}}^{m}$ denote the temperature-dependent thermal conductivity and specific heat, respectively, evaluated at temperature $T_{N_{D}}^{m}$. The density is denoted by $\rho$ and is constant. Solving Eq. (5) yields the heat flux history at node $n=N_{D}$, or equivalently, at depth $x=x_{2}$. 


\section{Solving for Temperature Heat Flux Histories Between Depths $x_{2}$ and $x=L$ (Inverse Problem)}

The region extending from thermocouple depth $x=x_{2}$ to the external surface $x=L$ is treated as the IHCP. The temperature and heat flux distributions are calculated in the domain $x \in\left[x_{2}, L\right]$ using a mesh of $N_{I}$ nodes where node $n=1$ is at depth $x=x_{2}$ and node $n=N_{I}$ is at $x=L$. The method used to solve the inverse problem is the space marching scheme S6 as outlined by Carasso ${ }^{5}$. The scheme has a truncation error of $O\left(\Delta t^{2}\right)$ and $O(\Delta x)$, and the scheme was found by Carasso to have the smallest amplification factor. Therefore, the S6 scheme was the most stable of the $O\left(\Delta t^{2}\right)$ schemes analyzed. The S6 scheme is derived by writing the 1-D heat conduction equation as a first-order system for the heat flux:

$$
q=k(T) \frac{\partial T}{\partial x}, \quad \frac{\partial q}{\partial x}=\rho c_{p}(T) \frac{\partial T}{\partial t}
$$

The temperature and heat flux at node 1 are known since $x_{2}$ is a shared boundary between the domain of the direct and inverse problems. Equation (6) is discretized using forward differencing in space and central differencing in time. For each node, $n=1, \ldots, N_{I}-1$ :

$$
\begin{array}{lll}
T_{n+1}^{0}=T_{n}^{0} & \text { and } & q_{n+1}^{0}=q_{n}^{0} \\
T_{n+1}^{m}=T_{n}^{m}+\Delta x\left(q_{n}^{m} / k_{n}^{m}\right) & \text { and } & q_{n+1}^{m}=q_{n}^{m}+\Delta x \rho c_{p_{n}}^{m}\left(T_{n}^{m+1}-T_{n}^{m-1}\right) / 2 \Delta t, \quad m=1, \ldots, M-1 \\
T_{n+1}^{M}=2 T_{n+1}^{M-1}-T_{n+1}^{M-2} & \text { and } & q_{n+1}^{M}=2 q_{n+1}^{M-1}-q_{n+1}^{M-2}
\end{array}
$$

Equations (7) to (9) yield temperature and heat flux distributions in the domain $x \in\left[x_{2}, L\right]$ marching from node 1 to node $N_{I}$ using boundary values given by the DHCP solution at depth $x_{2}$, i.e.

$$
T\left(x_{2}, t\right)=T_{\mathrm{DHCP}, x_{2}} \quad \text { and } \quad q\left(x_{2}, t\right)=q_{\mathrm{DHCP}, x_{2}}
$$

where $T_{\mathrm{DHCP}, x_{2}}$ denotes the filtered temperature at depth $x_{2}$ and $q_{\mathrm{DHCP}, x_{2}}$ denotes the heat flux solution at depth $x_{2}$ calculated from Eq. (5). Starting with node 1, the solution is marched in time before preceding to the next node.

\section{Methodology Characterization}

The methodology in Pizzo, et. al, ${ }^{1}$ is characterized by studying (a) the effects of embedded thermocouple depth through the thickness of a material on the accuracy of the estimated surface temperature and heat flux, (b) the sensitivity of the surface solution to the error in the specified location of the embedded thermocouples, and (c) the sensitivity to the error in the thermocouple data. For the characterization, it is assumed that one thermocouple is installed on the back face of a material and a second thermocouple is embedded through the thickness. The motivation for this work is to study the factors that affect solution accuracy and identify best practices when solving inverse problems using the methodology presented.

\section{Model Problem}

Each numerical study is performed using a model problem for which an analytical solution exists. The model assumes that one thermocouple is embedded through the thickness of a material and a second thermocouple is installed on the back face. Numerical solutions are compared to the exact analytical solution to assess accuracy of the method.

Assuming constant thermophysical properties, the heat conduction equation (1) reduces to:

$$
\frac{1}{\alpha} \frac{\partial T}{\partial t}=\frac{\partial^{2} T}{\partial x^{2}}
$$

where $\alpha=k / \rho c_{P}$. In each study, Eq. (11) is solved analytically in $0 \leq x \leq L$ for $t \in[0,100] \mathrm{s}$, with initial and boundary conditions:

$$
\begin{aligned}
& T(x, 0)=294 \mathrm{~K}, \quad 0 \leq x \leq L \\
& k(\partial T / \partial x)(0, t)=q_{0}, \quad t>0 \\
& k(\partial T / \partial x)(L, t)=q_{L}, \quad t>0
\end{aligned}
$$


and thermophysical properties:

$$
k=0.0575 \mathrm{~W} /(\mathrm{m}-\mathrm{K}), \quad \rho=0.0015 \mathrm{~kg} / \mathrm{cm}^{3}, \quad c_{P}=1833.33 \mathrm{~J} /(\mathrm{kg}-\mathrm{K}) .
$$

Boundary conditions of $q_{0}=0 \mathrm{~W} / \mathrm{cm}^{2}, q_{L}=40 \mathrm{~W} / \mathrm{cm}^{2}$ (herein identified as the Constant Case) and $q_{0}=0 \mathrm{~W} / \mathrm{cm}^{2}$, $q_{L}=(40-\mathrm{t} / 10) \mathrm{W} / \mathrm{cm}^{2}$ (herein identified as the Linear Case) are considered in Eq. (12). The imposed heat flux boundary conditions at depth $x_{L}$ are graphed versus time in Figure 2 for both the (a) Constant Case and (b) Linear Case, where $t \in[0,100] \mathrm{s}$. The corresponding analytical temperature solutions at depth $x_{L}$, denoted by $T_{L}$, are graphed versus time in Figure 3 for both the (a) Constant Case and (b) Linear Case.

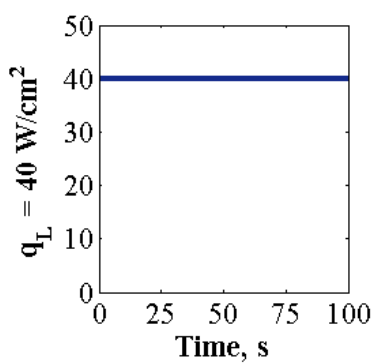

(a) Constant Case

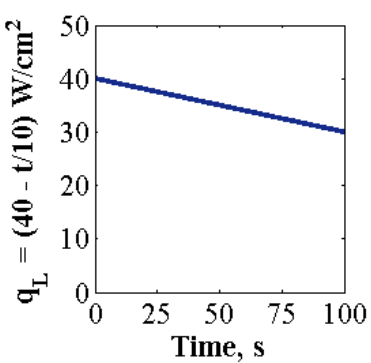

(b) Linear Case

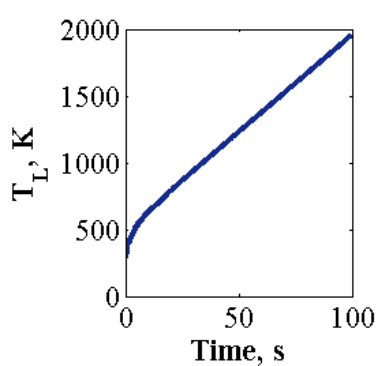

(a) Constant Case

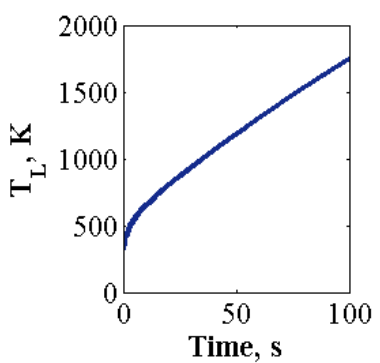

(b) Linear Case

Figure 2. Illustration of the imposed heat flux $q_{L}$ graphed versus time.

Figure 3. Illustration of the analytical temperature $T_{L}$ graphed versus time.

For each of the numerical studies presented in this paper, solution convergence was achieved by refining the spatial domain of the direct problem. With each successive refinement, the cell size was divided in half and the unbiased variance was calculated between consecutive refinements using the temperature of the node at the midpoint in the spatial domain. Once the variance fell below $0.01 \mathrm{~K}$, the solution was considered converged using the refinement of the smaller cell size. Moreover, time-steps of $\Delta t=0.1 \mathrm{~s}$ and $\Delta t=0.01 \mathrm{~s}$ were considered in each study.

The estimated and exact temperature at $x=L$ are used to compute the error in temperature:

$$
e_{T}(t)=100\left(\frac{\text { estimated } T(L, t)-T_{L}}{T_{L}}\right)
$$

where the exact temperature $T_{L}$ is obtained analytically. The graphical representations of the analytical temperatures are shown in Figure 3. The estimated and imposed heat flux at $x=L$ are used to compute the error in heat flux:

$$
e_{q}(t)=100\left(\frac{\text { estimated } q(L, t)-q_{L}}{q_{L}}\right)
$$

where the imposed heat flux is given by $q_{L}=40 \mathrm{~W} / \mathrm{cm}^{2}$ in the Constant Case and $q_{L}=(40-\mathrm{t} / 10) \mathrm{W} / \mathrm{cm}^{2}$ in the Linear Case. The graphical representations of the imposed heat flux are shown in Figure 2. The absolute values were removed from the standard error calculation in Eqs. (14) and (15) to examine when the numerical solution either overestimated $(e>0)$ or underestimated $(e<0)$ the solution.

\section{Effects of Embedded Thermocouple Depth Through the Thickness of a Material}

A numerical study was conducted to determine if there is an optimal depth to embed one thermocouple through the thickness of a material. Fifteen different depths were considered for the location of the embedded thermocouple, namely depths $x_{1}, x_{2}, \ldots x_{14}, x_{15}$ as illustrated in Figure 4. Using the analytical temperature solutions of Eqs. (11) to (13) at depth $x_{0}$ paired with the solutions at depths $x_{1}, x_{2}, \ldots x_{14}, x_{15}$ as inputs to the DHCP, heat flux histories are estimated at depths $x_{1}, x_{2}, \ldots x_{14}, x_{15}$. The boundary values in Eq. (10) are then given by the analytical temperature and estimated heat flux at each depth, and are used to solve the IHCP to estimate the surface temperature and heat flux histories, $T_{L}$ and $q_{L}$, respectively. 


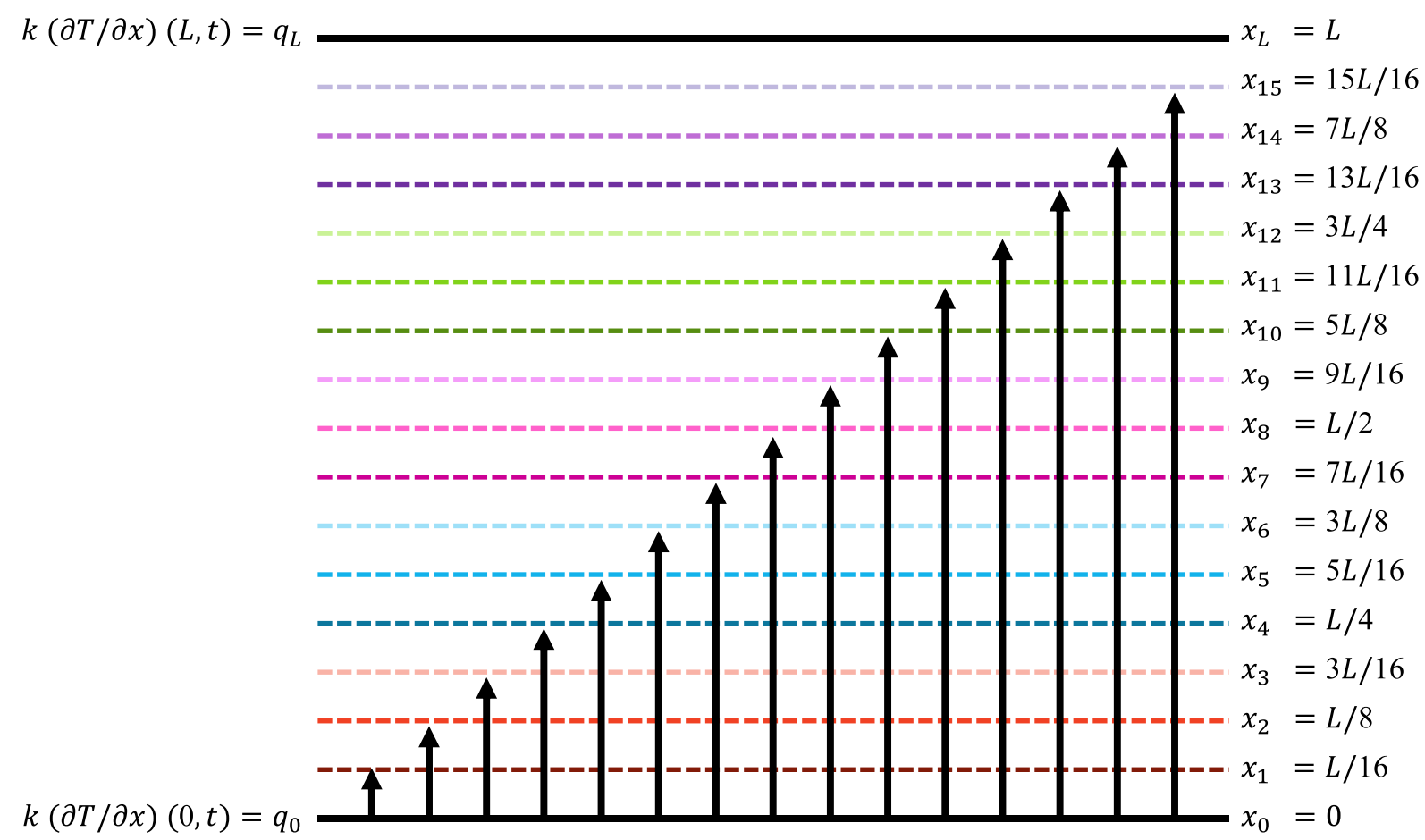

Figure 4. Illustration of the fifteen different depths considered for the embedded thermocouple.

In both the Constant and Linear Case studies with time-step of $\Delta t=0.01 \mathrm{~s}$, the ill-posedness of the inverse problem resulted in growing oscillations as the solution marched from each simulated thermocouple depth to $x_{L}$. The oscillatory growth was greatest for depths $x_{1}$ to $x_{6}$, yielding meaningless results with embedded thermocouples nearest the back face. The oscillations are a direct result of the domain size in the inverse problem combined with a small time-step. With small time-steps, larger domain sizes for the inverse problem allow for greater oscillatory growth. On the other hand, in both the Constant and Linear Case studies with time-step of $\Delta t=0.1 \mathrm{~s}$, the effect of the illposedness of the inverse problem was negligible for all embedded thermocouple depths $x_{1}$ to $x_{15}$. Temperature and heat flux estimations are therefore only given for the Constant and Linear Case studies with time-step of $\Delta t=0.1 \mathrm{~s}$. Moreover, only the estimations from depths $x_{7}$ to $x_{15}$ are considered for assessment due to the growing oscillations observed for depths $x_{1}$ to $x_{6}$ with the smaller time-step of $\Delta t=0.01 \mathrm{~s}$.

The Constant Case temperature and heat flux estimates are shown in Figure 5(a) and Figure 5(b) for $\Delta t=0.1 \mathrm{~s}$. Neglecting depths $x_{1}$ to $x_{6}$, the smallest magnitude of error in temperature is obtained for depths $x_{8}, x_{11}, x_{12}, x_{7}$, and $x_{10}$ with error ranging from $0 \%$ to $4 \%$ between $t \in[0,10] \mathrm{s}$, and from $0 \%$ to $2 \%$ by $t=100 \mathrm{~s}$. The largest magnitude of error in temperature is obtained for depths $x_{13}, x_{15}, x_{9}$, and $x_{14}$ with error greater than $5 \%$ between $t \in[0,10] \mathrm{s}$, and from $2 \%$ to $3 \%$ by $t=100 \mathrm{~s}$. The smallest magnitude of error in heat flux is obtained for depths $x_{12}, x_{11}, x_{10}, x_{7}$, $x_{13}$, and $x_{15}$ with error ranging from $-1 \%$ to $1 \%$ for $t \in[0,100] \mathrm{s}$. The largest magnitude of error in heat flux is obtained for depths $x_{9}, x_{8}$, and $x_{14}$ with error greater than $\pm 2 \%$ for $t \in[0,100] \mathrm{s}$.

The Linear Case temperature and heat flux estimates are shown in Figure 6(a) and Figure 6(b) for $\Delta t=0.1 \mathrm{~s}$. Neglecting depths $x_{1}$ to $x_{6}$, the smallest magnitude of error in both temperature and heat flux is obtained for depths $x_{15}, x_{14}, x_{13}, x_{12}, x_{11}$, and $x_{10}$ with error decreasing below $-0.2 \%$ by $t=100 \mathrm{~s}$ for temperature, and decreasing below $0.05 \%$ by $t=100 \mathrm{~s}$ for heat flux.

In the Linear Case, depths $x_{13}$ to $x_{15}$ yield the smallest magnitude of error in both temperature and heat flux, followed closely by depths $x_{10}$ to $x_{12}$. However, in the Constant Case, depths $x_{13}$ to $x_{15}$ yield the largest magnitude of error in both temperature and heat flux, whereas depths $x_{10}$ to $x_{12}$ yield the smallest. Considering all of the numerical results, the studies demonstrate that when using the methodology presented in [1] the surface estimations for both temperature and heat flux are consistently the most accurate if one thermocouple is embedded between depths $x_{10}$ and $x_{12}(5 L / 8$ and $3 L / 4)$ when the second thermocouple is installed on the back face. This finding is based entirely off of the model problem previously discussed, and is independent of time-step. Further assessments should be conducted to conclusively make this assertion, as alluded to in the concluding remarks in Section IV. 


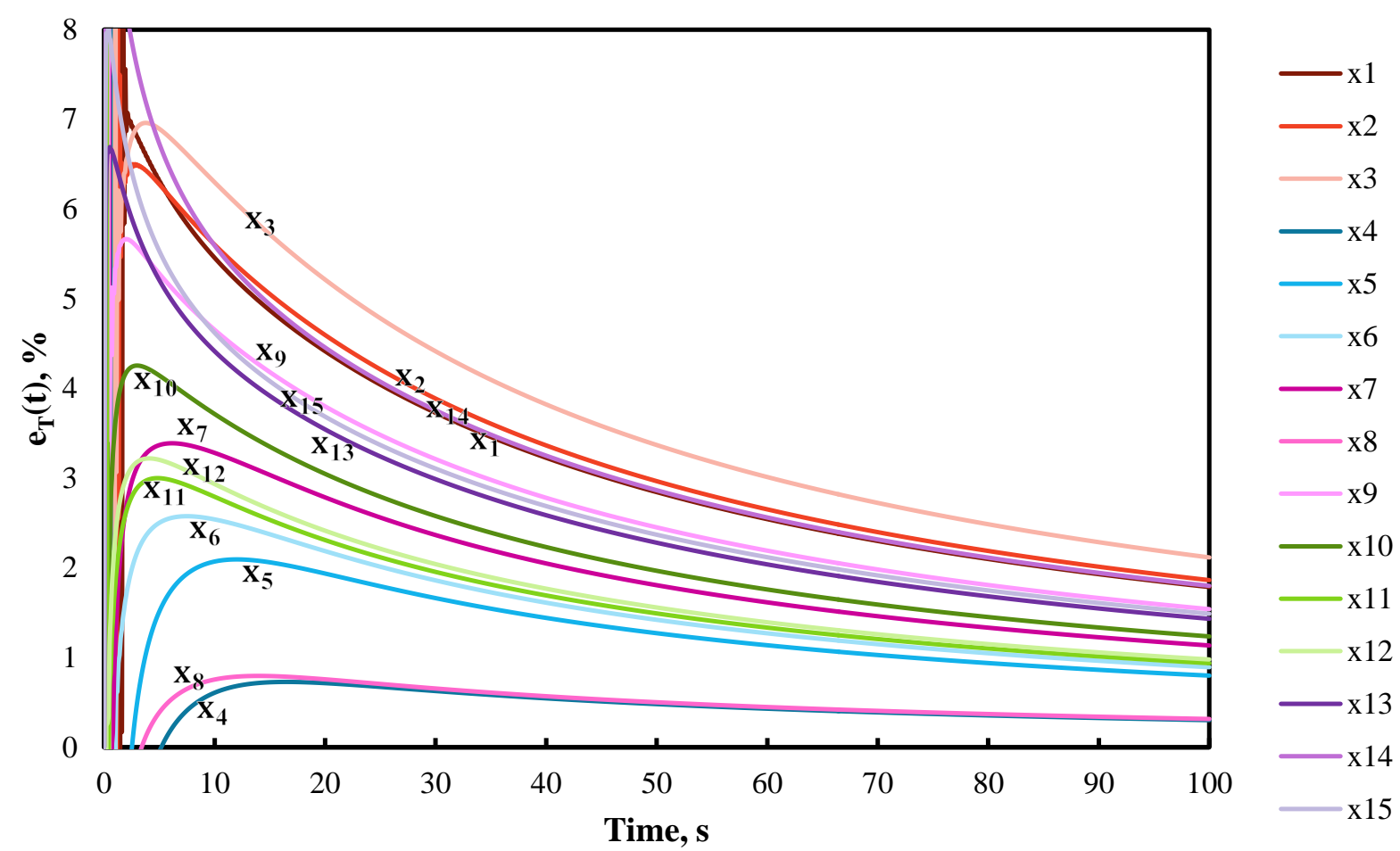

(a) Temperature error at $x=L$
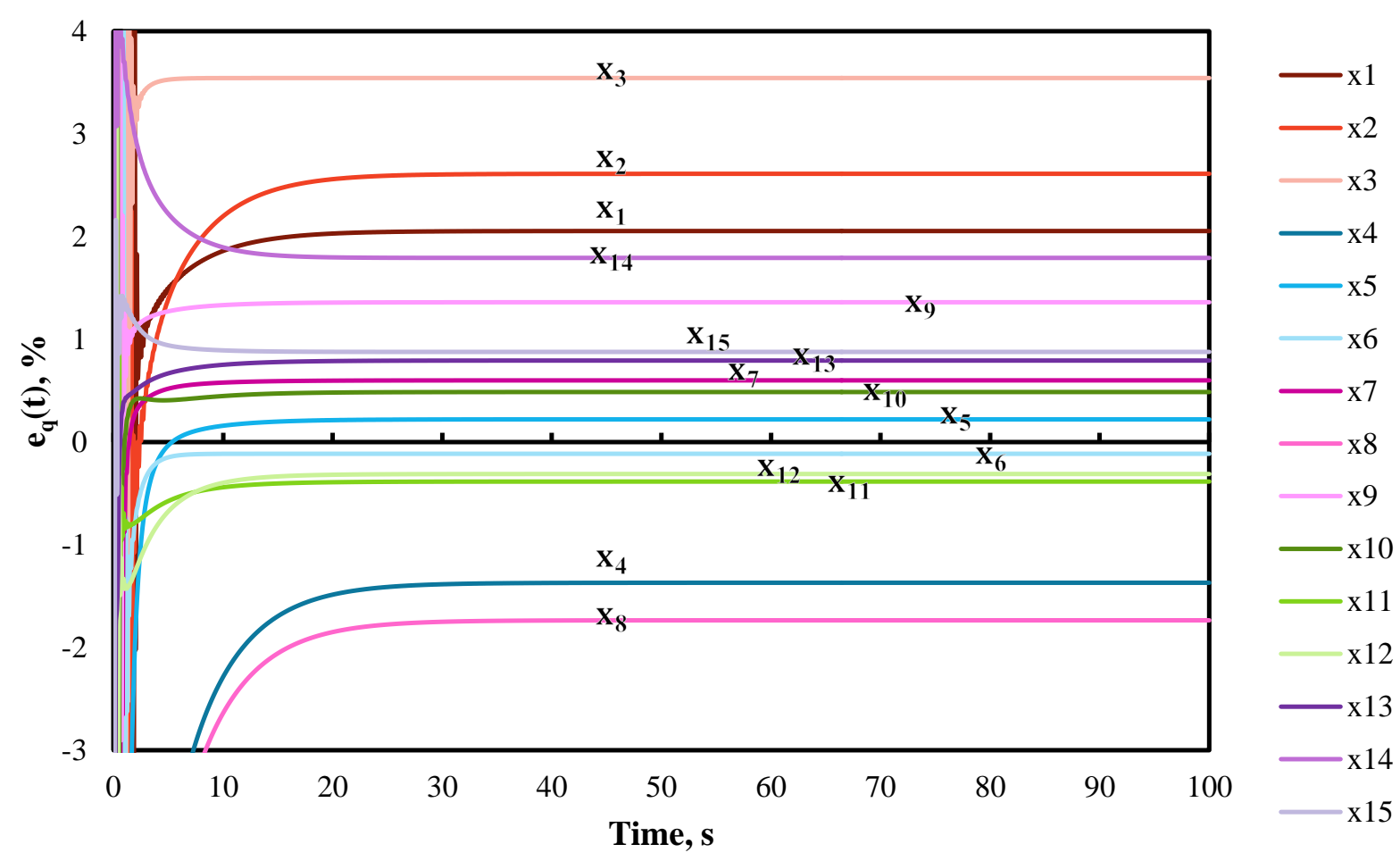

(b) Heat flux error at $x=L$

Figure 5. Error in the Constant Case estimations using a time step of $\Delta t=0.1 \mathrm{~s}$. 


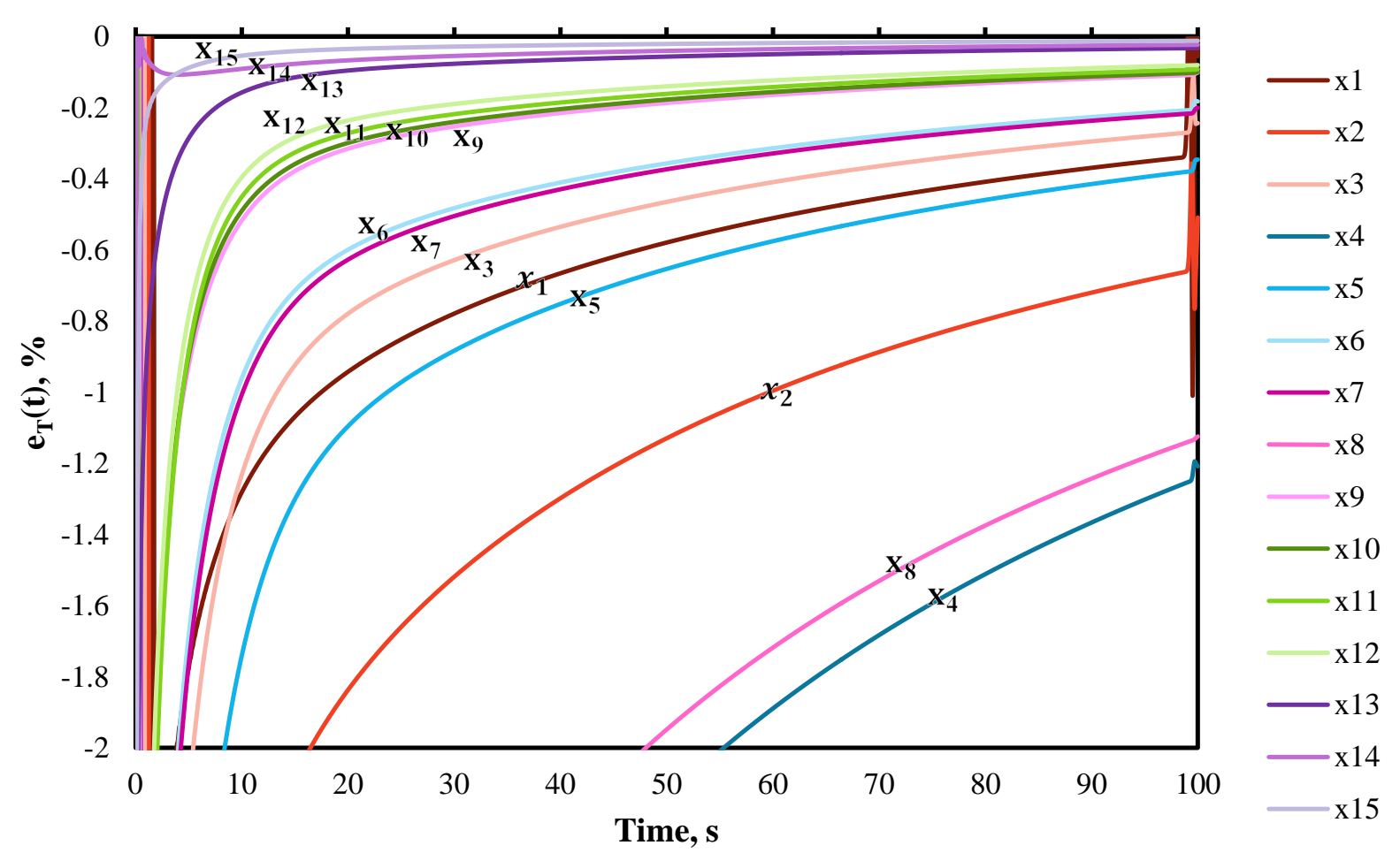

(a) Temperature error at $x=L$

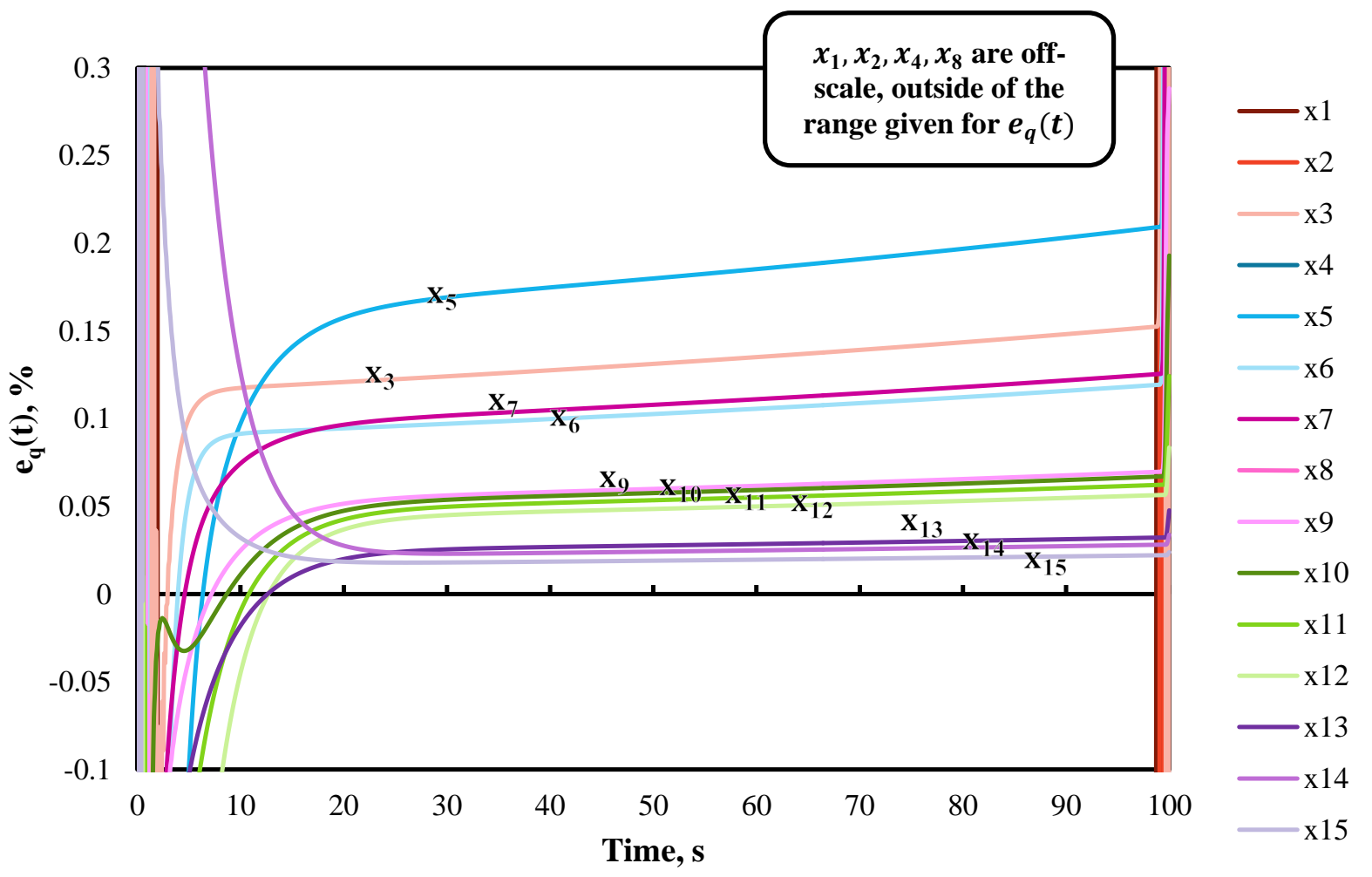

(b) Heat flux error at $x=L$

Figure 6. Error in the Linear Case estimations, comparing the numerical results to the analytical solution with considering time-step of $\Delta t=0.1 \mathrm{~s}$. 


\section{Sensitivity of Surface Solution to Error in Specified Location of the Embedded Thermocouple}

A numerical study was conducted to assess the sensitivity of the surface solution to the error in the specified location of the embedded thermocouple. For the sensitivity study, the embedded thermocouple is assumed to be at depth $3 L / 4$. The depth of $3 L / 4$ was selected because the previous results demonstrate that the estimations for both temperature and heat flux are consistently the most accurate when the embedded thermocouple is between depths $5 L / 8$ and $3 L / 4$.

To simulate error in the specified location of the thermocouple, the stated methodology is solved with accurate temperature data located at inaccurate depths. The embedded depth is perturbed by a factor of $\delta$. For $\delta=0.01,0.02$, $0.03,0.04,0.05,0.10$, and 0.15 , the DHCP is solved with analytical solutions of $T\left(x_{1}, t\right)$ and $T\left(x_{2} \pm \delta L, t\right)$ and depth inputs of $x_{1}=0$ and $x_{2}=3 \mathrm{~L} / 4$. The solution yields $q_{\mathrm{DHCP}, x_{2}}$ defined by Eq. (10). Boundary values $T\left(x_{2} \pm \delta L, t\right)$ and $q_{\mathrm{DHCP}, x_{2}}$ are then used to solve the IHCP to estimate the surface histories, $T_{L}$ and $q_{L}$.

Surface estimations are compared against the error in temperature and heat flux, $e_{T}$ and $e_{q}$ respectively, obtained from the embedded thermocouple depth study. The known $e_{T}$ and $e_{q}$ from depth $x_{12}$, shown in Figure 5 and Figure 6 , are used as a baseline in which case the perturbation, $\delta$, is equal to 0.00 . Since the model problem is a linear problem, the difference in error in the estimated temperature and heat flux is expected to be proportional to the prescribed error in the simulated baseline data.

The Constant Case temperature and heat flux estimates are shown in Figure 7(a) and Figure 7(b) for $\Delta t=0.1 \mathrm{~s}$. Additionally, the Linear Case temperature and heat flux estimates are shown in Figure 8(a) and Figure 8(b) for $\Delta t=$ 0.1 s. Results demonstrate that for both the Constant and Linear Case, as $\delta$ increases (decreases) towards +0.15 $(-0.15)$, the error in surface temperature and heat flux increases (decreases). Hence, the difference in error in the estimated temperature and heat flux is proportional to the prescribed baseline error, which was to be expected. The results also demonstrate that the heat flux error bounds remain constant with time whereas the temperature error bounds decrease with time. Moreover, while the error in temperature stays well within the bounds of the perturbation $( \pm 15 \%)$, the error in heat flux follows closely to the bounds of the perturbation.

\section{Sensitivity of Surface Solution to Error in Thermocouple Data}

A numerical study was also conducted to assess the sensitivity of the surface solution to the error in thermocouple data. For the sensitivity study, the embedded thermocouple is assumed to be at depth $3 L / 4$.

To simulate error in thermocouple data, the stated methodology is solved with inaccurate temperature data located at accurate depths. The temperature data are perturbed by a factor of $\delta$. For $\delta=0.01,0.02,0.03,0.04,0.05,0.10$, and 0.15 , the DHCP is solved with analytical solutions of $T\left(x_{1}, t\right)$ and $T\left(x_{2}, t\right) \pm \delta T\left(x_{2}, t\right)$ and depth inputs of $x_{1}=0$ and $x_{2}=3 \mathrm{~L} / 4$. The solution yields $q_{\mathrm{DHCP}, x_{2}}$ defined by Eq. (10). Boundary values $T\left(x_{2}, t\right) \pm \delta T\left(x_{2}, t\right)$ and $q_{\mathrm{DHCP}, x_{2}}$ are then used to solve the IHCP to estimate the surface histories, $T_{L}$ and $q_{L}$.

Surface estimations are compared against the error in temperature and heat flux, $e_{T}$ and $e_{q}$, respectively, obtained from the embedded thermocouple depth study. The known $e_{T}$ and $e_{q}$ from depth $x_{12}$, shown in Figure 5 and Figure 6 , are used as a baseline in which case the perturbation, $\delta$, is equal to 0.00 . Since the model problem is a linear problem, the difference in error in the estimated temperature and heat flux is expected to be proportional to the prescribed error in the simulated baseline data.

The Constant Case temperature and heat flux estimates are shown in Figure 9(a) and Figure 9(b) for $\Delta t=0.1 \mathrm{~s}$. Additionally, the Linear Case temperature and heat flux estimates are shown in Figure 10(a) and Figure 10(b) for $\Delta t=0.1$ s. Results demonstrate that for both the Constant and Linear Case, as $\delta$ increases (decreases) towards +0.15 $(-0.15)$, the error in surface temperature and heat flux increase (decrease). Hence, the difference in error in the estimated temperature and heat flux is proportional to the prescribed baseline error, which was to be expected. The results also demonstrate that the temperature error bounds remain constant with time, whereas the heat flux error increases with time. Moreover, while the error in temperature follows closely to the bounds of the perturbation $( \pm 15 \%)$, the error in heat flux is well outside the bounds of the perturbation. 


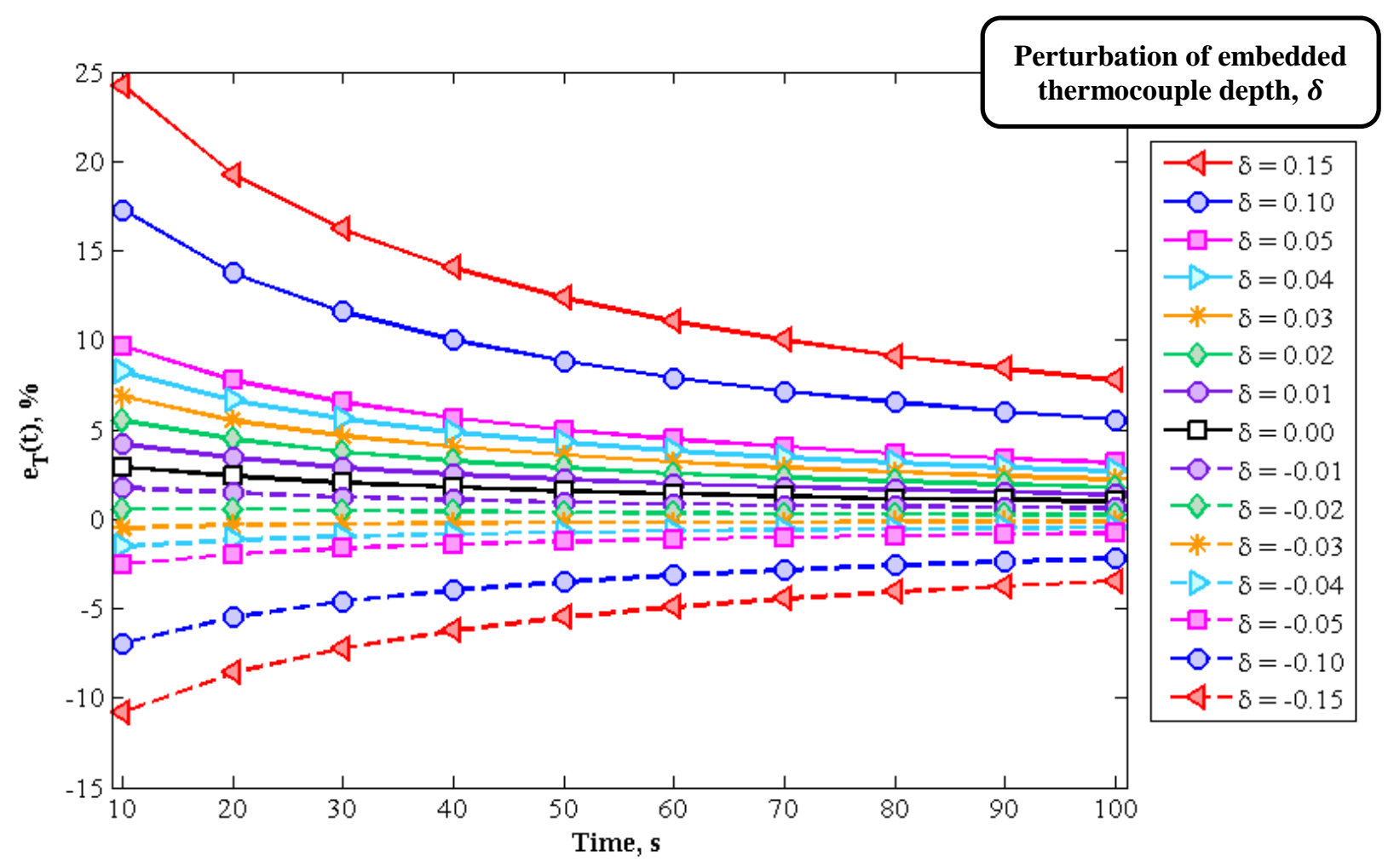

(a) Temperature error at $x=L$

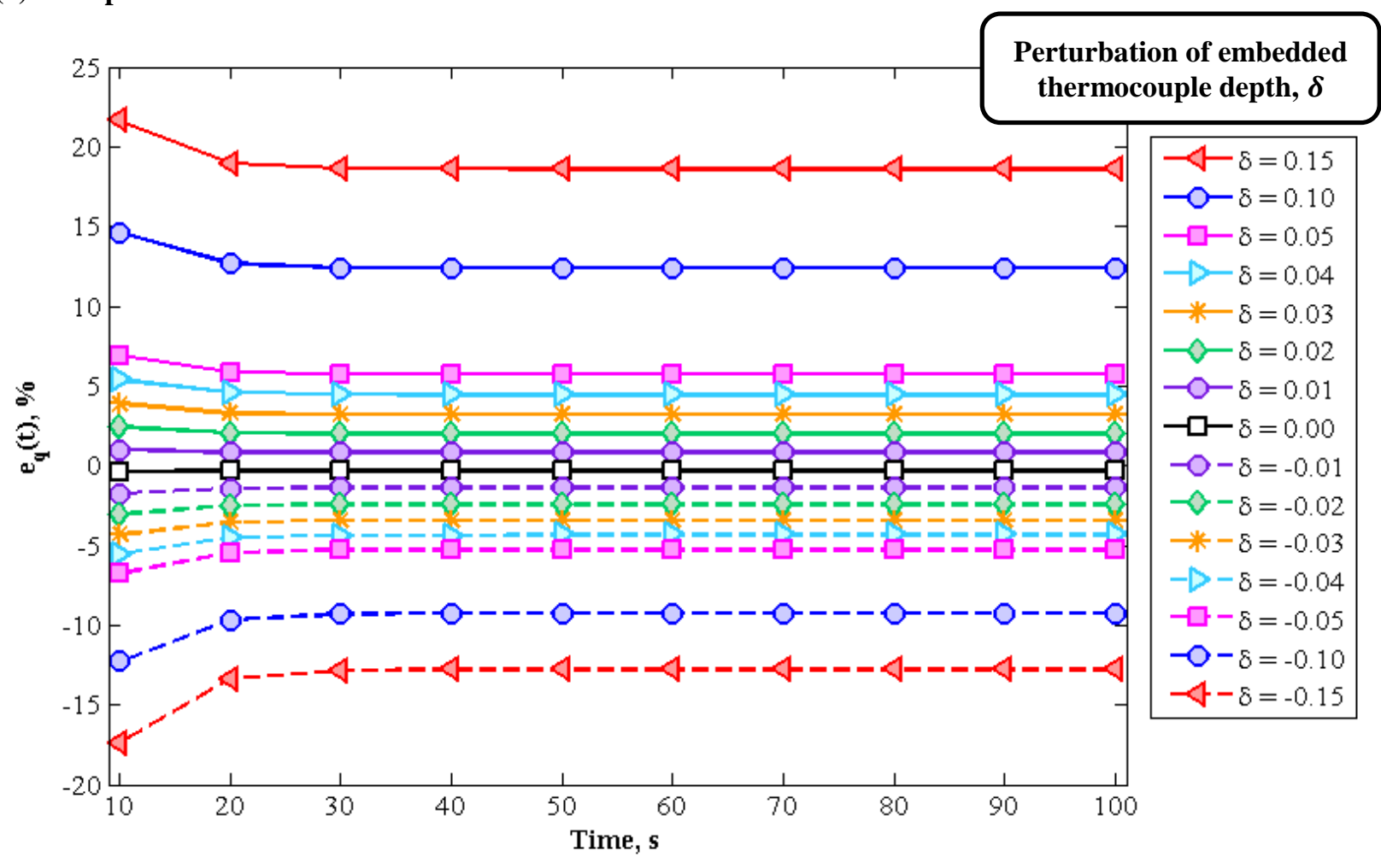

(b) Heat flux error at $x=L$

Figure 7. Sensitivity of the surface estimations to the error in the specified location of the embedded thermocouple for the Constant Case using a time-step of $\Delta t=0.1 \mathrm{~s}$. 


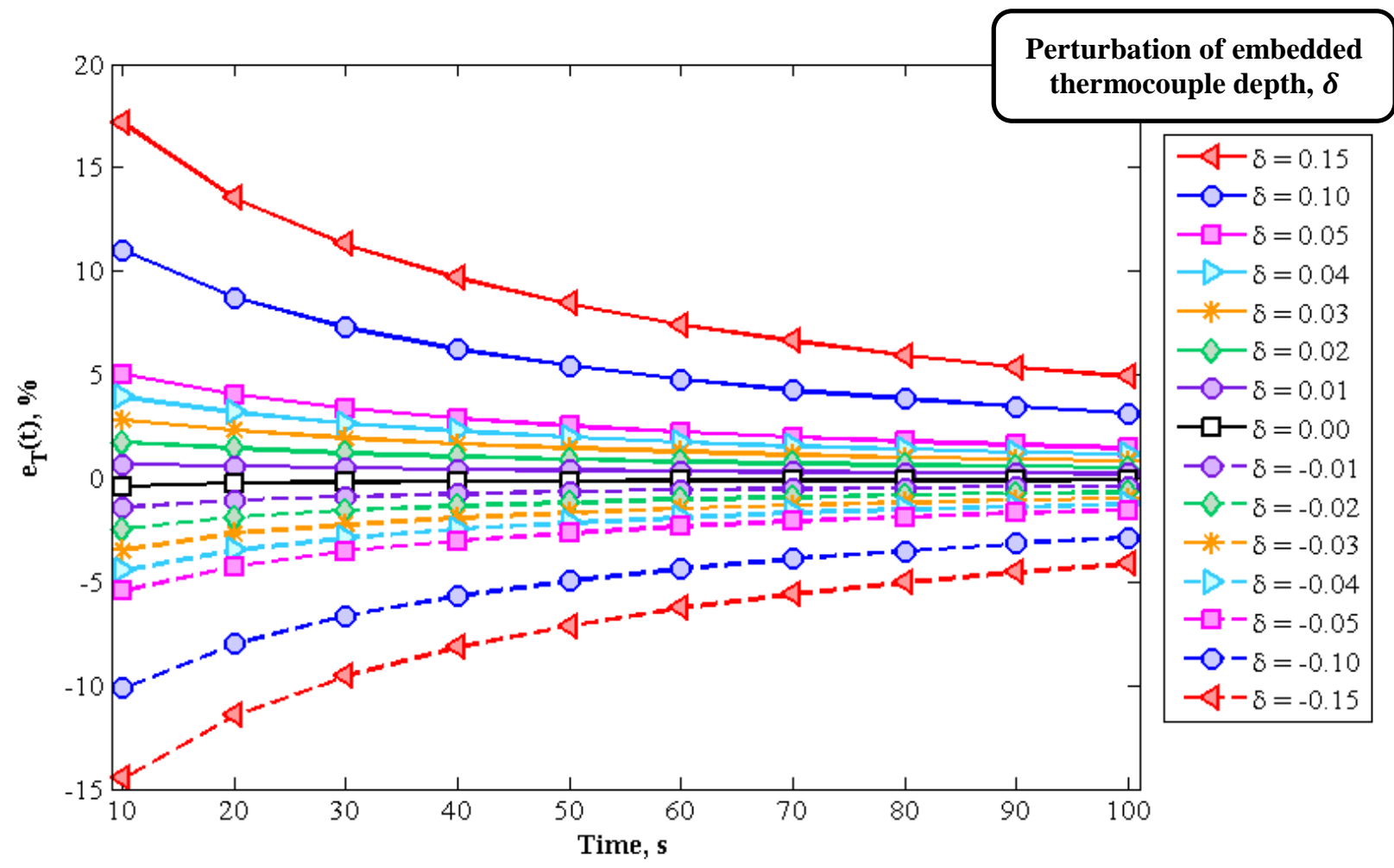

(a) Temperature error at $x=L$

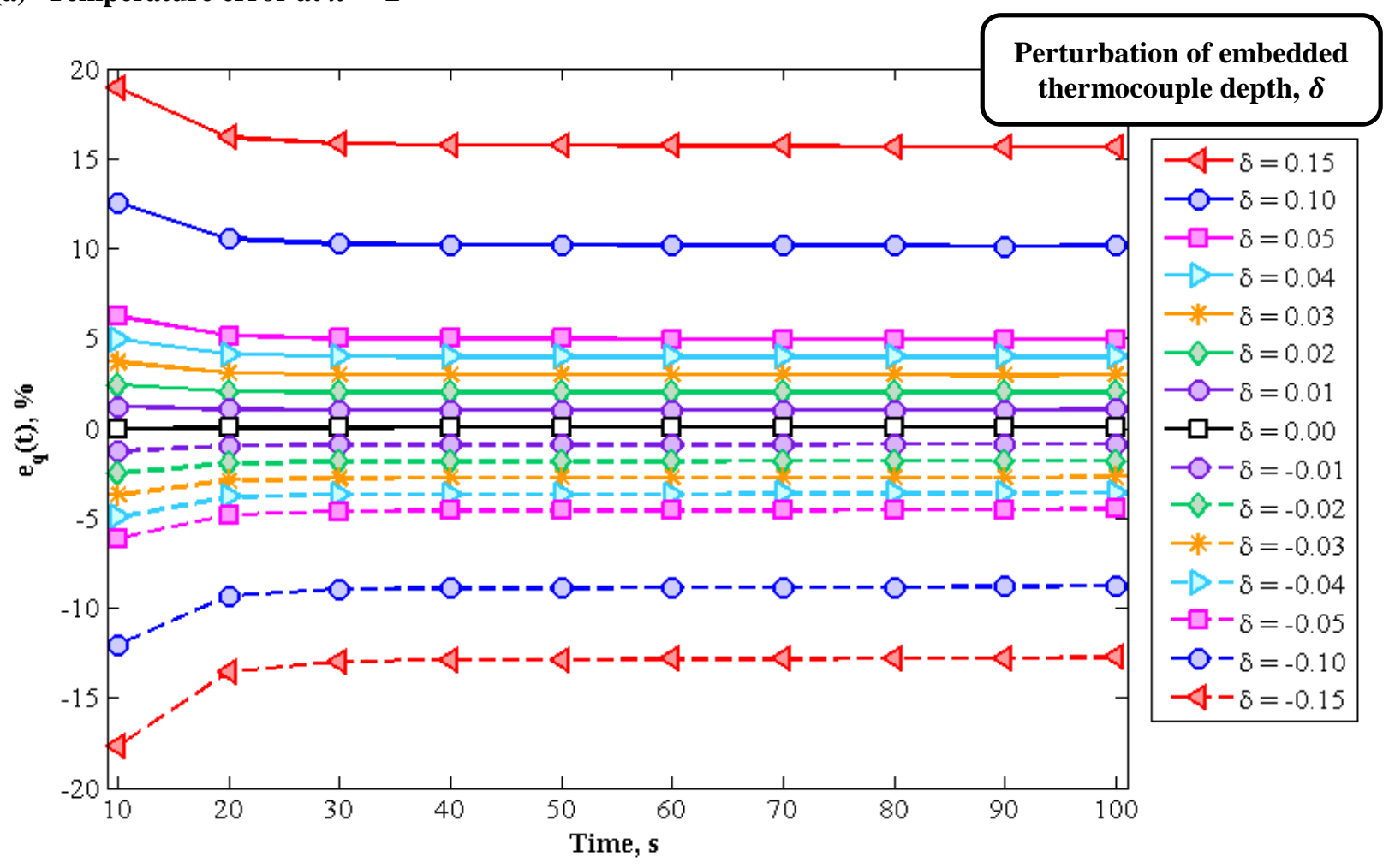

(b) Heat flux error at $x=L$

Figure 8. Sensitivity of the surface estimations to the error in the specified location of the embedded thermocouple for the Linear Case using a time-step of $\Delta t=0.1 \mathrm{~s}$. 


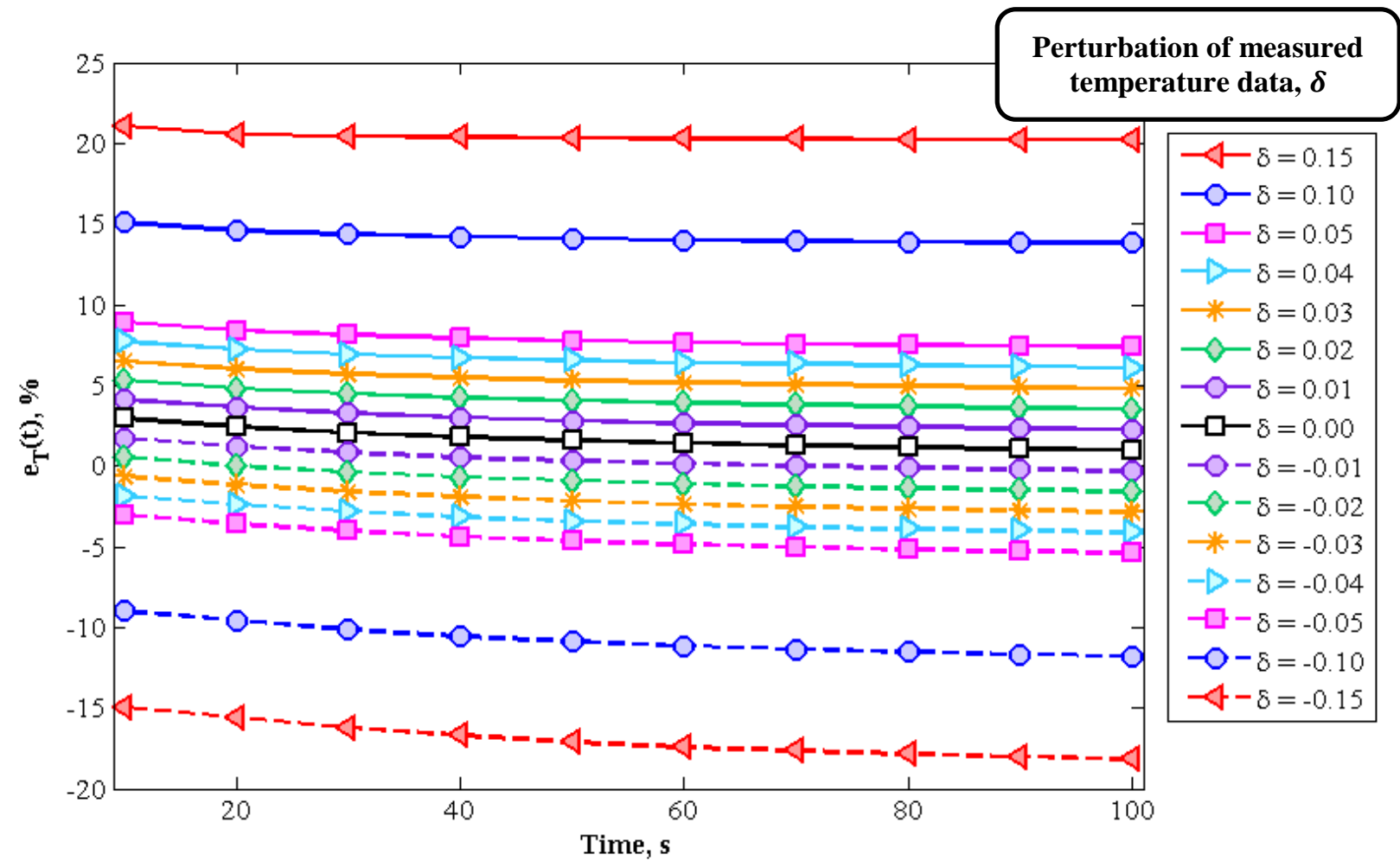

(a) Temperature error at $x=L$

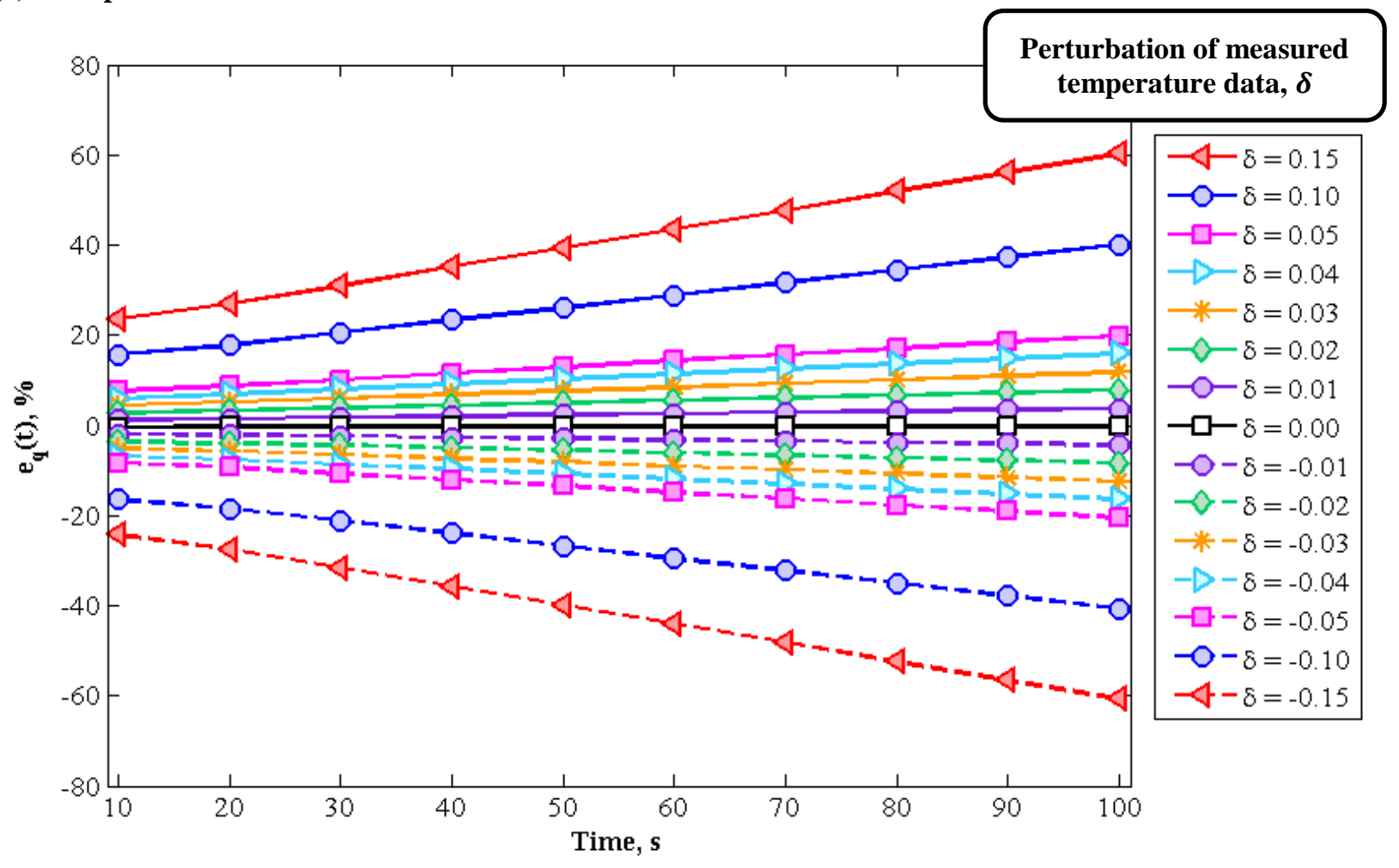

(b) Heat flux error at $x=L$

Figure 9. Sensitivity of the surface estimations to the error in thermocouple data for the Constant Case using a time-step of $\Delta t=0.1 \mathrm{~s}$. 


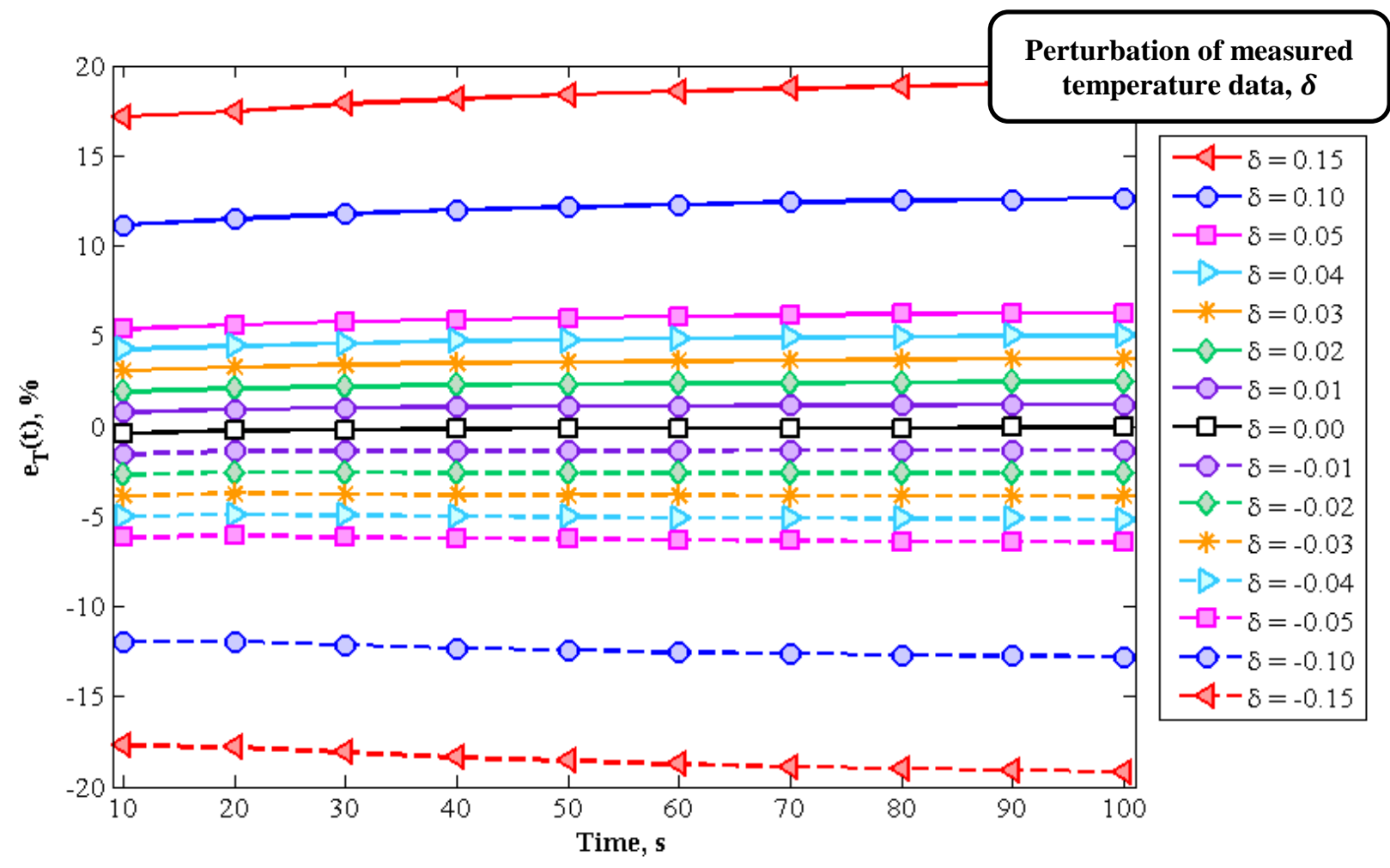

(a) Temperature error at $x=L$

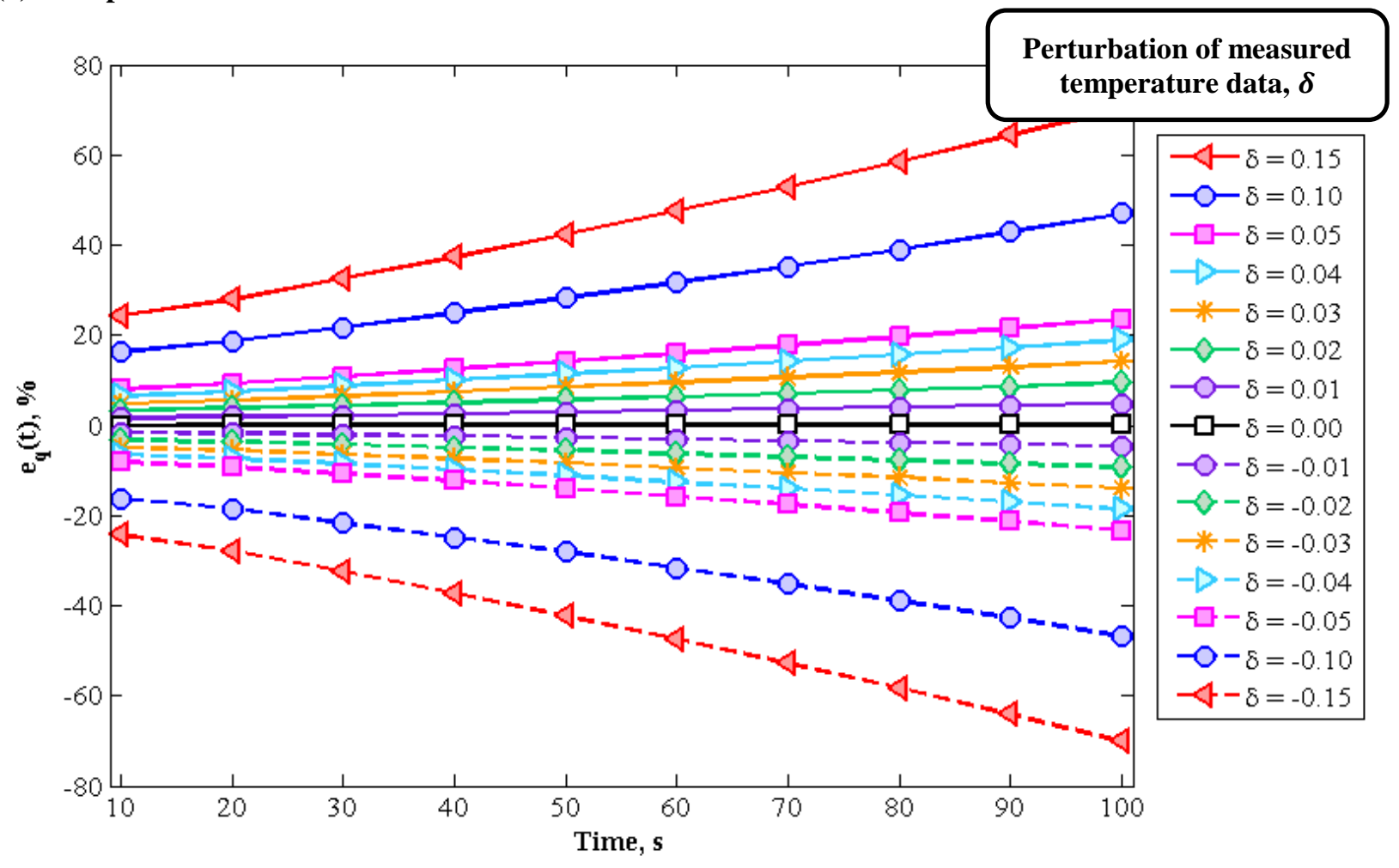

(b) Heat flux error at $x=L$

Figure 10. Sensitivity of the surface estimations to the error in thermocouple data for the Linear Case using a time-step of $\Delta t=0.1 \mathrm{~s}$. 


\section{Concluding Remarks}

The purpose of this paper is to expand upon the 2016 work of Pizzo, et. al, ${ }^{1}$ by characterizing the selected method for inverse heat conduction. Due to the ill-posedness of inverse problems, small errors in the data yield large errors in the solution. When solving inverse problems, accuracy in the estimated surface temperature and heat flux is dependent on several factors. Factors include the embedded thermocouple depth through the thickness of a material, the sensitivity of the surface solution to the error in the specified location of the embedded thermocouples, and the sensitivity to the error in thermocouple data. The effect of these factors on solution accuracy is presented in this paper. Each numerical study is performed using a model problem. The model assumes that one thermocouple is embedded through the thickness of a material and a second thermocouple is installed on the back face. Numerical data are compared against an exact analytical solution.

Preliminary results of the study to determine if there is an optimal depth to embed one thermocouple through the thickness of a material demonstrate that the surface estimations for both temperature and heat flux are consistently the most accurate if one thermocouple is embedded between depths $x_{10}$ and $x_{12}(5 L / 8$ and $3 L / 4)$ when a second thermocouple is installed on the back face. This finding is based entirely off of the model problem, and is independent of time-step. Further assessments should be conducted to conclusively make this assertion, by perhaps considering a radiative boundary condition for $q_{L}$ with temperature-dependent thermophysical properties. This type of study would better emulate the testing conditions of carbon/carbon materials. Test data can then be used to validate the numerical findings. To assess whether the optimal depth is dependent on either the physical or numerical aspects of the problem, other spatial divisions should also be considered, e.g. by dividing the domain from $x=0$ to $x=L$ into perhaps 20 or 100 evenly spaced intervals.

When assessing the sensitivity of the surface solution to (a) the error in the specified location of the embedded thermocouple and to (b) the error in thermocouple data, the difference in error in the estimated temperature and heat flux is proportional to the prescribed baseline error, which was to be expected. The results also demontrate that when assessing the error in the specified location of the embedded thermocouple, the heat flux error bounds remain constant with time whereas the temperature error bounds decrease with time and when assessing the error in thermocouple data, the temperature error bounds remain constant with time, whereas the heat flux error increases with time. This disparity between sensitivity assessments is also to be expected.

When perturbing the embedded depth, the thermocouple temperature remains unchanged from the baseline problem. In this case, the estimated surface temperature and heat flux are merely offset by a factor of the perturbation and no additional heat is added to, or removed from, the system. Hence, the results of depth perturbation follows closely to the baseline results of temperature [error decreases with time as indicated in Figure 5(a) and Figure 6(a)] and heat flux [error remains constant with time as indicated in Figure 5(b) and Figure 6(b)]. On the other hand, when perturbing the thermocouple data, additional heat is added to $(+\delta)$ or removed from $(-\delta)$ the baseline problem. With the addition (removal) of heat, the surface estimations for both temperature and heat flux will increase (decrease) with time as compared to the baseline problem. Hence, rather than the error in temperature decreasing with time as is the case for the baseline problem, the additional heat added/removed from the system causes the error to remain constant. Similarly, rather than the error in heat flux remaining constant with time as is the case for the baseline problem, the additional heat added/removed from the system causes the error to increase. This result demonstrates that any error in embedded depth will translate to a proportional error of the same order of magnitude, and any error in thermocouple data will translate to a proportional error of a higher order of magnitude. Therefore, when a user instruments a material with two thermocouples and post-processes the measured data to estimate the surface temperature and heat flux, it is of utmost importance that the measured depths and temperature data are accurate.

\section{Acknowledgments}

The authors would like to thank Dr. Kim Bey and Dr. Kamran Daryabeigi of NASA Langley Research Center. Dr. Bey played a vital role in teaching the first author the skills necessary to conduct this research. Her technical expertise and guidance were unparalleled and the first author is extremely grateful for Dr. Bey's mentorship. Moreover, Dr. Daryabeigi's insight and leadership were instrumental in the first author's ability to learn the field of heat transfer and inverse methods, and are greatly appreciated.

The first author would additionally like to thank the LARSS (Langley Aerospace Research Summer Scholars) and NIFS (NASA Internships, Fellowships, and Scholarships) programs for providing the opportunity to intern at NASA Langley Research Center. These two programs were paramount to the first author being able to learn from and be mentored by her extremely knowledgeable co-author Dr. David E. Glass. 


\section{References}

${ }^{1}$ Pizzo, M. E., Glass, D. E., and Bey, K.S., "Analysis of Internal Thermocouple Data in Carbon/Carbon Using Inverse Heat Conduction Methods," 54 th AIAA Aerospace Sciences Meeting, San Diego, California, AIAA SciTech, (AIAA 2016-0508).

${ }^{2}$ Weber, C. F., "Nonlinear Estimation Applied to the Nonlinear Inverse Heat Conduction Problem," Int. J. Heat Mass

Transfer, Vol. 13, 1970, pp. 703-716.

${ }^{3}$ Beck, J. V., Blackwell, B., and St. Clair Jr., C. R., Inverse Heat Conduction: Ill-Posed Problems, Wiley, New York, 1985.

${ }^{4}$ Frankel, J. I., "Regularization of Inverse Heat Conduction by Combination of Rate Sensor Analysis and Analytic Continuation," J. Eng. Math, 2007, pp. 57, 181-198.

${ }^{5}$ Carasso, A. S., "Space Marching Difference Schemes in the Nonlinear Inverse Heat Conduction Problem," Inverse Problems, Vol. 8, No. 1, 1992, pp. 25-43.

${ }^{6}$ Murio, D.A, "The Mollification Method and the Numerical Solution of the Inverse Heat Conduction Equation by Finite Differences," Computers Math. Applic., Vol. 17, No. 10, 1989, pp. 1385-1396.

${ }^{7}$ Shumakov, N. V., "A Method for the Experimental Study of the Process of Heating a Solid Body," Sov. Phys. Tech, Phys. (translated by American Institute of Physics), Vol. 2, 1957, pp. 771-781.

${ }^{8}$ Stolz, Jr. G., "Numerical Solutions to an Inverse Problem of Heat Conduction for Simple Shapes," J. Heat Transfer, Vol. 82, 1960, pp. 20-26.

${ }^{9}$ Beck, J. V., "Surface Heat Flux Determination Using an Integral Method," Nuclear Engineering and Design, Vol. 7, No. 2, 1968, pp. 170-178.

${ }^{10}$ Beck, J. V., "Nonlinear Estimation Applied to the Nonlinear Inverse Heat Conduction Problem," Int. J. Heat Mass

Transfer, Vol. 13, 1970, pp. 703-716.

${ }^{11}$ Beck, J. V., Blackwell, B., Haji-Sheikh, A., "Comparison of Some Inverse Heat Conduction Methods using Experimental Data," Int. J. Heat Mass Transfer, Vol. 39, No. 17, 1996, pp. 3649-3657.

${ }^{12}$ Frankel, J. I. and Arimilli, R. V., "Inferring Convective and Radiative Heating Loads from Transient Surface Temperature Measurements in the Half-Space," Inverse Problems in Science and Engineering, Vol. 15, No. 5, 2007, pp. 463-488.

${ }^{13}$ Frankel, J. I. and Keyhani, M., "Moving Low-Pass Gauss Filter with Automatically Defined Influence Region for Diffusive Studies," J. Thermophysics, Vol. 26, No. 1, 2012, pp. 176-181.

${ }^{14}$ Blosser, M. L., Daryabeigi, K., Bird, R. K., and Knutson, J. R., “Transient Thermal Testing and Analysis of a Thermally Insulating Structural Sandwich Panel,” NASA TM-2015-218701, NASA Langley Research Center, Hampton, Virginia, 2015. 